\title{
New Modified English and Hindi Oswestry Disability Index in Low Back Pain Patients Treated Conservatively in Indian Population
}

\author{
Nishant ${ }^{1}$, Harvinder Singh Chhabra ${ }^{2}$, Kulwant Singh Kapoor ${ }^{3}$ \\ ${ }^{1}$ Department of Spine Services, Rameshwaram Orthopedic and Spine Clinic, Patna, India \\ ${ }^{2}$ Department of Spine Services, Indian Spinal Injuries, New Delhi, India \\ ${ }^{3}$ Department of Biostatistics, All India Institute of Medical Science, New Delhi, India
}

Study Design: Prospective cohort study along with questionnaire.

Purpose: To measure the correlation of the visual analogue score (VAS), with (Oswestry disability Index [ODI], version 2.1a) in English, and modified ODI (English and Hindi version). To validate translated version of the modified ODI in English version to Hindi.

Overview of Literature: Conflicting evidence in literature regarding the ability for existing ODI score to accurately measure the pain associated disability.

Methods: One hundred and three patients conservatively treated for low back pain were enrolled in the study. The Pearson correlation coefficient for VAS and ODI along with the Cronbach $\alpha$ and test-retest reliability for Hindi version using the intraclass correlation coefficient was recorded. The new proposed translated Hindi version of ODI was carried out with established guidelines.

Results: The mean age in English and Hindi version of ODI was 53.5 years and 58.5 years, respectively. The gender ration was 21:24 in the English version and 35:23 in the Hindi version. The mean follow-up in English and Hindi version of ODI was 3.4 months and 50.27 months, respectively. The Cronbach coefficient $\alpha=0.7541$ for English ODI and 0.9913 for Hindi ODI was recorded for the both modified versions.

Conclusions: The new modified ODI is time saving and accurate, and it avoids the need to measure other scores and has stronger correlation with VAS score compared to the previous scores. We recommend this version for both English and Hindi speaking population as an assessment tool to measure the disability related to pain.

Keywords: Back pain; Oswestry disability index; Outcome measures; Validity; Reliability; Indian population

\section{Introduction}

The original Oswestry disability index (ODI), developed by Fairbank et al. [1], consists of ten items that assess the level of pain and interference with several physical activities: sleeping, self-care, sex life, social life, and traveling. Two of the most commonly used disability scales for people with lower back pain (LBP) are the Roland-Morris Disability Scale and the Oswestry Low Back Pain Disability Questionnaire (OSW) [1-4]. These self-reported measurements have been developed for people with LBP, and their importance as measures of treatment outcome in clinical trials has been emphasized [3]. A recent study by Deyo et al. [3] recommended the Roland-Morris Dis-

Received Oct 19, 2013; Revised Jan 14, 2014; Accepted Feb 4, 2014

Corresponding author: Nishant

Rameshwaram Orthopedic \& Spine Clinic, K 79 P.C. Colony Hanuman Nagar, Kankarbagh, Patna-800020, Bihar, India

Tel: +91-95-2551-7801, Fax: +91-61-2235-7137, E-mail: nishantspine@gmail.com 
ability Questionnaire (RMDQ) or the Oswestry Disability Questionnaire (ODI) for measure of back pain related outcome. These qustionnaires have been translated into various languages and followed the guidelines laid by Beaton et al. [4] for the process of cross-cultural adaptation of self-report measures. These outcome measures are usually classified as disease specific [5], generic [6-12], or common generic measures [6-8] and uses the Medical Outcomes Survey Short-Form 36 (SF-36) [9,10]. Several measures are available for assessing functional outcomes related to low back pain [5,11-22]. However, in our experience, the present scores have poor correlation between the pain (VAS score) and the associated disability (ODI score); this result is, probably because they measure different parameters. It becomes important for a clinician to assess the degree of disability due to pain among these individuals in order to guide the treatment or improvement with the on-going measures to improve outcome. The RMDQ has tried to make it more specific by adding the phrase "because of my back $[18,19]$." In our part of the world, the majority of the patients in urban areas are fluent in English and Hindi. India is multicultural, a diverse with people who speak different languages. Hindi is one of the national languages in India, spoken in the majority of the states, and is the most common language among such a diverse country. The validity and reliability of English and another Indian language, Marathi, are already established [23]. The objective of this study was to test the validity and reliability of the new modified ODI in Hindi for patients with LBPs in New Delhi. This study has also attempted to simplify the assessment of disability related only to pain. If the disability was related to other causes, then the score was not taken into account. We used the current form of ODI (version 2.1a) with this modification because we, the authors, believe that the modified ODI was simpler and less time taking and had better correlation with the VAS score and do not require health-related quality of life (HRQoL) outcomes.

\section{Materials and Methods}

The original and new modifications of the ODI were done by a spine fellow and chief of spine services. The inclusion criteria were all patients conservatively treated with low back pain. Exclusion criteria included infection, tumour, patients unable to follow the scoring system, and those patients who were not bilingual. The questionnaires were repeated both in English and Hindi and recorded. Any disparity was noted in the comprehension. After 103 patients with low back pain were referred to the authors' department and had completed the questionnaire (Version 2.1a and the modified version), they were interviewed by the principal investigator, who probed each patient about their thought of the questionnaire. One modification was made to the questionnaire for all the sections. If the patient disability was not due to pain, then score of zero was given. Patients participating in the final test were recruited on the basis of the following criteria, as described below.

\section{Forward translation}

The forward translation was carried out by two bilingual individuals who were proficient in both languages (English and Hindi), in speaking and writing. The first translator, also the first author of this article, is a spine surgeon. The other is a professional translator, with no medical background. The purpose of the translation was not disclosed to the professional translator. The two translated versions of the modified English version (original English version of ODI 2.1a) were compared and discussed with the two translators and the spine services chief professor; this comparision and discussion continued until a synthesis of the translation was reached.

\section{1) Back translation}

The back translations were completed by two bilingual translators, with Hindi as their mother tongue, but were professional translational services in the English language. They independently translated the synthetic version into English. Both translators had no medical background and were not informed or aware of the prior translation procedure. A team of all the authors and professional Hindi translators was formed, discussed all the versions of the translation, along with the original version, and reached a consensus on a single version and did so without any discrepancies. Following the previous guidelines, a pre-test version was created on the basis of the synthetic forward translation. A pilot study was conducted to test whether the translated version was understood correctly by Indian (Hindi language speaking) patients. Forward and backward translations were done. Patients participating in the final test were recruited on the basis of the following criteria, as descibed below. 
2) Inclusion criteria

(1) History of LBP for at least two days; (2) Diagnosis of LBP confirmed by a spine surgeon and/or an orthopaedic surgeon; and (3) Age above eighteen years.

\section{3) Exclusion criteria}

(1) Patients who had already received spine surgery or whose low back pain was caused by a recent external injury. (2) Pregnant and lactating females were also excluded from this study. We followed established guidelines for the processes of cross cultural adaptation of self-report measures, the translatio, $n$ and adaptation process with ODI (version 2.1a). The final version (Hindi) was obtained from the pre-test version and tested for its validity and reliability together with the VAS $(10 \mathrm{~cm})$ score.

\section{4) Test-retest}

After the first test, patients were retested with the Hindi version of the modified ODI within one to two weeks at the follow-up. No special intervention, except for the patient's routine treatment at the outpatient clinic, was carried out.

\section{5) Sample size}

By conducting a survey, 103 patients of both sexes were recruited from an outpatient Spine Service clinic at a tertiary care hospital and after getting approval from institutional ethics Committee, during April 2013 to June 2013. ODI is a self-administered questionnaire divided into ten sections, each with six items designed to assess limitations of various activities of daily living. Each section is scored on a 0 to 5 scale, with 5 representing the greatest disability. The index is calculated by dividing the summed score by the total possible score, which is then multiplied by 100 and expressed as a percentage. Thus, for every question not answered, the denominator is reduced by 5 . If a patient marked more than one statement for a question, the highest scoring statement is recorded as the true indication of disability. It takes 3.5 to 5 minutes to complete the questionnaire and approximately 1 minute to score. The scores were assessed from $0 \%$ to $20 \%$ to indicate minimal disability, $20 \%$ to $40 \%$, to indicate moderate disability, $40 \%$ to $60 \%$ to indicate severe disability, $60 \%$ to $80 \%$ to indicate crippled, and $80 \%$ to $100 \%$ to indicate bedbound or exaggerating [1].

\section{Patient recruitment}

Our centre is visited by patients from numerous states and follow-up is troublesome. Since financial constraints of patients coming from other cities were one of the issues, we selected patients who were resident of Delhi for an easy follow-up.

\section{Statistical analyses}

Descriptive statistics and socio demographic data were used to describe the samples, as illustrated in Table 1. The internal consistency of the instrument was examined with the cronbach $\alpha$. Correlation was calculated from the first (test) and second (retest) administration of the ODI of the Hindi version, only for the modified, and also between the original and modified ODI for English version. Test-retest reliability of the Hindi version of the modified ODI was tested by using the intraclass correlation coefficient. The Pearson correlation coefficient was used to evaluate criterion-related validity. The level of significance adopted for the statistical tests was $1 \%$, that is, $p \leq 0.01$. Reliability was estimated through internal consistency and stability (test-retest) assessment for the Hindi version.

\section{Results}

Out of 103 reviewed with low back pain that was treated conservatively with an average follow-up of 3.4 months 58.17 months for English and Hindi, respectively, version of the ODI. Forty-five patients were assessed with English version of the ODI, and 58 patients were assessed with the Hindi version of the modified version of the ODI. The mean age in English and Hindi version of ODI was 53.5 years and 58.5 years, respectively. The gender ratio was 21:24 (English version) and 35:23 in Hindi version, with only two patients married in the English version and all patients married in Hindi version (range, 21-86 years). The meaning of both the questionnaire and the responses were explored. All 103 patients correctly understood the questionnaire. However, only 34 patients in the Hindi version answered Section 8 (sex life) of the ODI. The response rate was very good $(100 \%$ for the first test and $100 \%$ for retest) for Hindi versions. In the English version, there were $47 \%$ male and $53 \%$ female participants while $42 \%$ of the participants had LBP for less than 
Table 1. Sociodemographic characteristics ( $n=103)$

\begin{tabular}{|c|c|c|c|}
\hline \multirow{2}{*}{ Variables } & \multicolumn{2}{|c|}{ English (n1= 45) } & \multirow{2}{*}{ Hindi (n2= 58) } \\
\hline & Previous ODI & Modified ODI & \\
\hline \multicolumn{4}{|l|}{ Sex } \\
\hline Male & 21 & 21 & 35 \\
\hline Female & 24 & 24 & 23 \\
\hline ODI modified & $17.06 \pm 13.3$ & - & 33.44/33.27 (test-retest) \\
\hline ODI previous version & $33.16 \pm 16.45$ & - & Not recorded \\
\hline Marital status & 43 & 43 & 58 \\
\hline Co-morbids & 9 & 9 & 15 \\
\hline Follow-up (mo) & 3.4 & - & 50.27 \\
\hline \multicolumn{4}{|l|}{ Age (yr) } \\
\hline$<30$ & 3 & 3 & 0 \\
\hline $30-49$ & 14 & 14 & 15 \\
\hline$>50$ & 28 & 28 & 43 \\
\hline Mean (standard deviation) & $53.49 \pm 14.4$ & $53.49 \pm 14.4$ & $58.15 \pm 12.09$ \\
\hline \multicolumn{4}{|l|}{ Duration of low back pain } \\
\hline$<1-3$ mo & 31 & 31 & 0 \\
\hline $3-6$ mo & 8 & 8 & 15 \\
\hline$>6 \mathrm{mo}$ & 5 & 5 & 43 \\
\hline \multicolumn{4}{|l|}{ ODI score } \\
\hline Minimal (0\%-20\%) & 12 & 33 & $1(1)$ \\
\hline Moderate $(21 \%-40 \%)$ & 16 & 9 & $45(45)$ \\
\hline Severe $(41 \%-60 \%)$ & 15 & 2 & $9(10)$ \\
\hline Crippled (61\%-80\%) & 2 & 1 & $1(0)$ \\
\hline Bed bound (81\%-100\%) & 0 & 0 & 0 \\
\hline
\end{tabular}

Values are presented as mean \pm standard deviation or number (\%).

ODI, Oswestry disability index.

3 months and the majority (85\%) of the participants had moderate to severe level of ODI scores. Pearson coefficient for correlation of the modifications and different version of ODI with VAS was significant with a $p$-value $<0.01$. Pearson correlation between initial VAS score of the English version and modified English version was 0.610 and 0.619 respectively, (for both $p$-value $<0.001$ ). VAS correlation with Hindi version was 0.984 (test and re-test) and highly significant with a $p$-value $<0.001$. The VAS correlation with original english in the original version was 0.610 and was also significant.

\section{Internal consistency (ICC)}

ICC for reliability was shown to have satisfactory results as indicated by the Cronbach Coefficient $\alpha=0.7541$ for the modified English version of ODI and 0.9913 for the Hindi version of the modified ODI. There was no significant difference between the mean ODI scores on the two test occasions (Fig. 1).

\section{Stability (test-retest)}

There was no significant difference between the mean ODI scores on the two test occasions for the Hindi versions. For the data obtained in the test and retest phases for 58 patients for the Hindi version, the stability could be tested and represented in Fig. 2. The English version test-retest was not performed. 


\section{Discussion}

Pain is a subjective symptom and differs on various factors in terms of perception, threshold, and the type of pain. There is growing consensus regarding clinicians that visual analogue score does not relate well with the ODI and this belief is probably because both measure

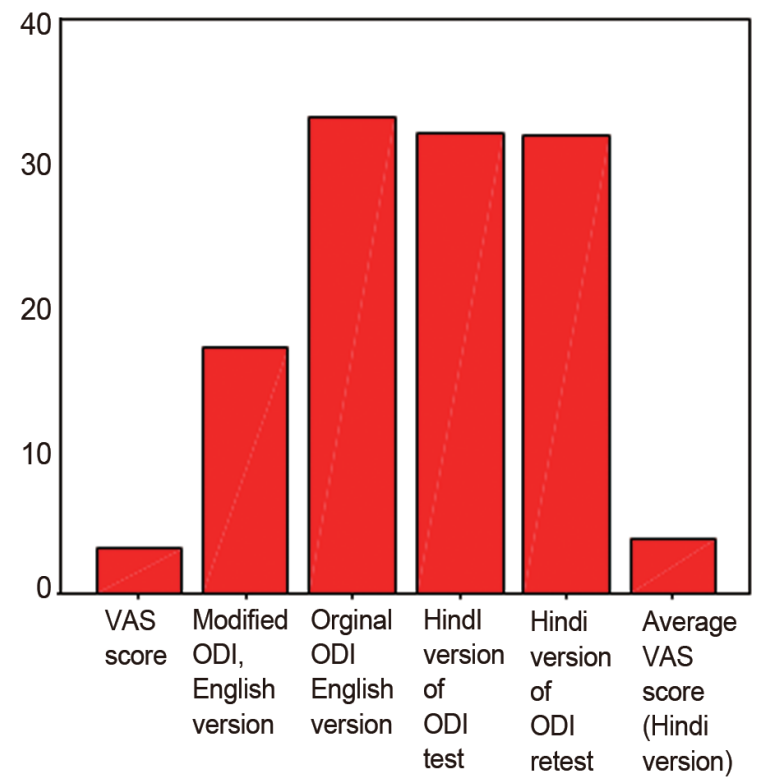

Fig. 1. A scatter plot for correlation: showing majority points falling close to a single straight line, indicating test-retest reliability of the Hindi version of the modification ODI. ODI, indicates Oswestry disability index; VAS, visual analogue score.

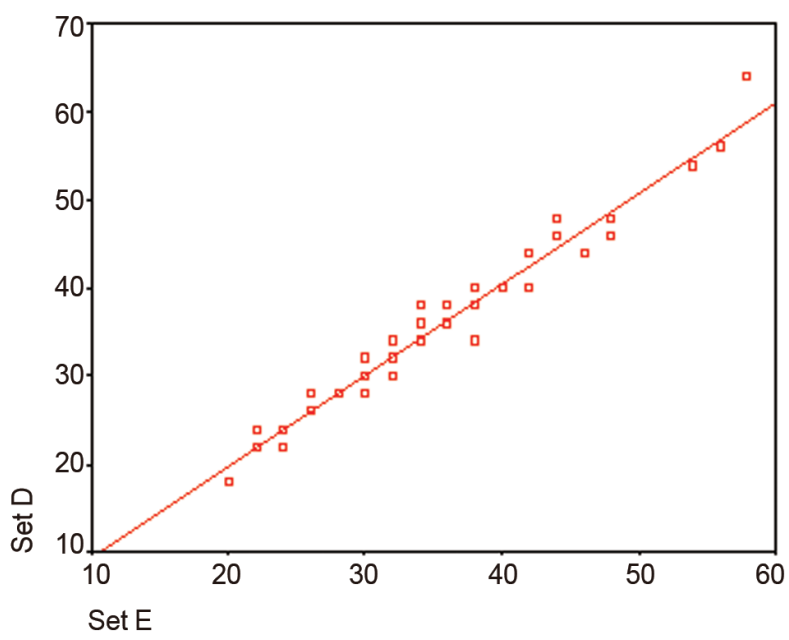

Fig. 2. A scatter plot for correlation: showing majority points falling close to a single straight line indicating test-retest reliability of Hindi version of the modification ODI. ODI, Oswestry disability index; Set D $\&$ Set $\mathrm{E}$, first test and retest, respectively. two different entities [24], despite a statically significant correlation as shown in our study and a previous study [23]. There is little correlation between the change in pain outcomes and the change HRQoL outcomes after spine surgery for low back pain [23]. At our institution, by using simple functional measurement tools for quick assessment of the patients' disability due to pain and response to conservative or operative treatment, we clinically and methodically evaluate the patient. It has been our clinical observation that, sometimes, the underlying causes for disability are factors other rather than pain, for example, stiffness, comorbid, and osteoporosis. Due to the availability of many scores for evaluation, it is difficult to effectively calculate pain-associated disability in a limited time and assess the functional outcome. The new modification to the existing ODI has been proposed for a quick, easy, and, yet, accurate assessment of the disabilityassociated pain. This article also illustrates the Indian population adaption of the frequently used for back pain specific ODI and presents results of psychometric testing. The items of translated Hindi showed both an excellent and full response rate. Internal consistency was excellent. Our translated version exceeded the previous reported Cronbachin English, which ranges between 0.71 and 0.87 [1].The English and Hindi versions of the ODI showed results similar to those presented in the original ODI English version with test-retest reliability [1,5,22], internal consistencies [1,5], and correlation with a VAS [22]. Many translated versions are available in foreign languages [25-28], and many Indian language, Marathi, versions [23] have been reported. The results of our study shows that our version of ODI had exceedingly better realtions of ODI with VAS score as compared to previously reported versions. To our knowledge, this Hindi version of the ODI is the first condition-specific outcome instrument for LBP in our subcontinent. The English ODI (modified version) and translated Hindi (modified version) ODI showed good internal consistency. The original English version and modified English version were tested with the same population. Once a stronger correlation of VAS with the modified ODI (English version) was established, the Hindi version of the modified ODI was tested and retested with an independent set of population for its reliability. This result is the first time that the modified ODI has been tested for Hindi version, which is the native language of India while English is a second language. The stability of the modified English version was not tested. 
This condition will not alter the results since, for Indian subcontinent, Hindi is the native language while English is the second language.

\section{Limitations}

We recruited only outpatients. Therefore, we could not compare the results with inpatients and, thus, discriminate validity could not be established, despite recording comorbidity. To ensure $100 \%$ follow-up, we selected patients who were resident of Delhi. Therefore, some degree of selection bias cannot be ruled out completely.

\section{Conclusions}

The results of this study indicate that the English as well as the translated Hindi versions of the ODI are reliable and valid instruments for the measurement of disability among Indian patients with LBP problems. We recommend these English and Hindi versions of the ODI for use in future clinical studies in India and the Hindi version with Hindi speaking patients with LBP. The stability of the modified English version of the modification is yet to be tested (Suppl Figs. 1, 2).

\section{Conflict of Interest}

No potential conflict of interest relevant to this article was reported.

\section{Supplementary Materials}

Suppl. Fig. 1. Oswestry disability index modified English version. Supplementary material can be found via http:// www.asianspinejournal.org/src/sm/asj-8-632-s001.pdf. Suppl. Fig. 2. Modified Oswestry disability index Hindi version. Supplementary material can be found via http:// www.asianspinejournal.org/src/sm/asj-8-632-s002.pdf.

\section{References}

1. Fairbank JC, Couper J, Davies JB, O’Brien JP. The Oswestry low back pain disability questionnaire. Physiotherapy 1980;66:271-3.

2. Deyo RA. Measuring the functional status of patients with low back pain. Arch Phys Med Rehabil 1988;69:1044-53.
3. Deyo RA, Battie M, Beurskens AJ, et al. Outcome measures for low back pain research. A proposal for standardized use. Spine (Phila Pa 1976) 1998;23:2003-13.

4. Beaton DE, Bombardier C, Guillemin F, Ferraz MB. Guidelines for the process of cross-cultural adaptation of self-report measures. Spine (Phila Pa 1976) 2000;25:3186-91.

5. Kopec JA, Esdaile JM, Abrahamowicz M, et al. The Quebec Back Pain Disability Scale. Measurement properties. Spine (Phila Pa 1976) 1995;20:341-52.

6. Patrick DL, Deyo RA. Generic and disease-specific measures in assessing health status and quality of life. Med Care 1989;27:S217-32.

7. Bergner M, Bobbitt RA, Carter WB, Gilson BS. The Sickness Impact Profile: development and final revision of a health status measure. Med Care 1981;19:787-805.

8. Hunt SM, McEwen J, McKenna SP. Measuring health status: a new tool for clinicians and epidemiologists. J R Coll Gen Pract 1985;35:185-8.

9. Parkerson GR Jr, Broadhead WE, Tse CK. The Duke Health Profile. A 17-item measure of health and dysfunction. Med Care 1990;28:1056-72.

10. McHorney CA, Ware JE Jr, Rogers W, Raczek AE, Lu JF. The validity and relative precision of MOS shortand long-form health status scales and Dartmouth COOP charts. Results from the Medical Outcomes Study. Med Care 1992;30:MS253-65.

11. Ware JE Jr, Sherbourne CD. The MOS 36-item shortform health survey (SF-36). I. Conceptual framework and item selection. Med Care 1992;30:473-83.

12. Bombardier C. Outcome assessments in the evaluation of treatment of spinal disorders: summary and general recommendations. Spine (Phila Pa 1976) 2000;25:3100-3.

13. Greenough CG, Fraser RD. Assessment of outcome in patients with low-back pain. Spine (Phila Pa 1976) 1992;17:36-41.

14. Kopec JA, Esdaile JM, Abrahamowicz M, et al. The Quebec Back Pain Disability Scale: conceptualization and development. J Clin Epidemiol 1996;49:151-61.

15. Manniche C, Asmussen K, Lauritsen B, Vinterberg H, Kreiner S, Jordan A. Low Back Pain Rating scale: validation of a tool for assessment of low back pain. Pain 1994;57:317-26.

16. Million R, Hall W, Nilsen KH, Baker RD, Jayson MI. 
Assessment of the progress of the back-pain patient 1981 Volvo Award in Clinical Science. Spine (Phila Pa 1976). 1982;7:204-12.

17. Roland M, Morris R. A study of the natural history of back pain. Part I: development of a reliable and sensitive measure of disability in low-back pain. Spine (Phila Pa 1976) 1983;8:141-4.

18. Ruta DA, Garratt AM, Wardlaw D, Russell IT. Developing a valid and reliable measure of health outcome for patients with low back pain. Spine (Phila Pa 1976) 1994;19:1887-96.

19. Waddell G, Main CJ. Assessment of severity in lowback disorders. Spine (Phila Pa 1976) 1984;9:204-8.

20. Williams RM, Myers AM. A new approach to measuring recovery in injured workers with acute low back pain: Resumption of Activities of Daily Living Scale. Phys Ther 1998;78:613-23.

21. Fujiwara A, Kobayashi N, Saiki K, Kitagawa T, Tamai K, Saotome K. Association of the Japanese Orthopaedic Association score with the Oswestry Disability Index, Roland-Morris Disability Questionnaire, and short-form 36. Spine (Phila Pa 1976) 2003;28:1601-7.

22. Gronblad M, Hupli M, Wennerstrand P, et al. Intercorrelation and test-retest reliability of the Pain Disability Index (PDI) and the Oswestry Disability Questionnaire (ODQ) and their correlation with pain intensity in low back pain patients. Clin J Pain 1993;9:189-95.

23. Joshi VD, Raiturker PP, Kulkarni AA. Validity and reliability of English and Marathi Oswestry Disability Index (version 2.1a) in Indian population. Spine (Phila Pa 1976) 2013;38:E662-8.

24. Fairbank JC, Pynsent PB. The Oswestry Disability Index. Spine (Phila Pa 1976) 2000;25:2940-52.

25. Lue YJ, Hsieh CL, Huang MH, Lin GT, Lu YM. Development of a Chinese version of the Oswestry Disability Index version 2.1. Spine (Phila Pa 1976) 2008;33:2354-60.

26. Jeon CH, Kim DJ, Kim SK, Kim DJ, Lee HM, Park HJ. Validation in the cross-cultural adaptation of the Korean version of the Oswestry Disability Index. J Korean Med Sci 2006;21:1092-7.

27. Lauridsen HH, Hartvigsen J, Manniche C, Korsholm L, Grunnet-Nilsson N. Danish version of the Oswestry Disability Index for patients with low back pain. Part 1: Cross-cultural adaptation, reliability and validity in two different populations. Eur Spine J 2006;15:1705-16.

28. Grotle M, Brox JI, Vollestad NK. Cross-cultural adaptation of the Norwegian versions of the RolandMorris Disability Questionnaire and the Oswestry Disability Index. J Rehabil Med 2003;35:241-7. 